\title{
Antimicrobial and larvicidal potential of endophytic fungi isolated from Tragia involucrata Linn.
}

Ananda Danagoudar, Chandrashekhar G. Joshi*, Nivya M.T., Manjunath H.M., Jagadeesha Poyya, Sunil Kumar R.

Department of Studies \& Research in Biochemistry, PG Centre, Mangalore University, Chikka Aluvara, Kodagu, Karnataka, India.

Received: November 16, 2016; Revised: November 23 2016; Accepted: December 17, 2016

\begin{abstract}
Endophytic fungi are one of the untapped resources of therapeutic compounds for various diseases. The present study focused on the antimicrobial as well as larvicidal activity of ethyl acetate extract of endophytic fungi isolated from Tragia involucrata. The ethyl acetate extract of Penicillium citrinum CGJ-C1 (GenBankNo.KT780618), Penicillium citrinumCGJ-C2 (KP739821), Cladosporiumsp. (KP739822), and Cryptendoxyla bypophloia CGJ-D2 (KT780619) was subjected to antimicrobial activity against a panel of microorganisms by disc diffusion method, larvicidal activity against Culex quinquefasciatus. All the extracts showed significant antimicrobial activity against the tested organisms ranging from $8 \pm 0.32$ to $13 \pm 2.11 \mathrm{~mm}$ (zone of inhibition). The extent of activity was comparable to the standard drugs. The larvicidal potential of the endophytes was superior to T. involucrata extract. The larvicidal activity was found to be dose and time dependent with $\mathrm{LC}_{50}$ value ranging from $4.25-158.06 \mathrm{ppm}$ after $24 \mathrm{hrs}$ of treatment. This is the first report on the bioactivity of the endophytes isolated from T. involucrata. Further studies on the bio-guided isolation of lead compound will benefit the people suffering from microbial diseases.
\end{abstract}

Key words: Tragia involucrata; Endophytic Fungi; Antimicrobial; Antiyeast; Larva.

\section{Introduction}

The demand for new antibiotic is escalating day by day due to the development of drug resistance even though many novel molecules are released to the market. Chemical synthesis and engineered biosynthesis are the major contributors to the several drugs. Although this method is extensively exploited for the new generic molecules, nature still astonishes us with the availability of versatile compounds. Even the chemical synthesis depends on the structure available in nature. So, an antibiotic discovery from nature is ever-increasing field of study (Supaphon et al., 2013).

Most of the rural people in the developing and under developing countries still depend on natural products or its derivatives for primary healthcare (Tamokou et al., 2013). Plants are the major providers of remedy for primary health associated disorders. They have been extensively used for treating various ailments since ages. Of late, researchers are focusing their attention on the myriads of untapped endophytic microorganisms symbiotically associated with plants (Cui et al., 2011). Endophytic fungi have the capability to produce the secondary metabolites similar to that of the host plant (Zhao et al., 2010). These microbes are reported to produce several therapeutic molecules which are studied against various ailments (Cui et al., 2011; Higginbotham et al., 2013; Strobel and Daisy, 2003). Endophytes have several advantages over the host plants in which they exist such as less complexity and easy to grow in mass culture. These properties make endophytes a new avenue for discovering novel drugs (Cui et al., 2011).

Tragia involucrata L. (Indian stinging nettle, Family Euphorbiaceae) is a medicinal plant having multifarious uses. Different parts of this plant has been reported to show cytotoxic (Joshi et al., 2011a), antitumor (Joshi et al., 2011b), antifertility (Joshi and Gopal, 2011), antimicrobial, larvicidal (Bhattacharya and Chandra, 2014), and antidiabetic (Farook and Atlee, 2011). Even though T. involucrata have been extensively studied for various medicinal properties, not even a single report was available on theisolation and biological properties of endophytes associated with this plant. This observation prompted us to isolate the endophytic fungi from $T$. involucrata and to study antimicrobial and larvicidal activity.

\section{Materials and Methods}

Chemicals

Potato dextrose agar (PDA), chloramphenicol, agarose, Sodium hypochlorite, ethyl acetate, potassium acetate and dimethyl sulfoxide (DMSO), were procured from Merck, India. All other chemicals used for the study were of analytical grade.

\section{Collection of plant material}

Fresh stem parts of T. involucrata were collected from in and around Mangalore, Karnataka, India (2013) in order

\footnotetext{
*Corresponding Author:

Dr. Chandrashekhar G. Joshi,

Department of Biochemistry,

Mangalore University, PG centre, Chikka Aluvara,

Thorenur post, Kushalnagarhobli, Somwarpet taluk,

Kodagu- 571 232, Karnataka, India.

E-mail: josheejoshee@yahoo.com
}

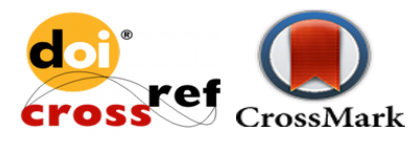


to study the endophytic fungi associated and transported to the lab in the sterile polyethylene bags. The Global Positioning System (GPS) locations were $12.5581^{\circ} \mathrm{N}$, $75.3892^{\circ} \mathrm{E}$.

\section{Isolation of endophytic fungi from $T$. involucrata}

Tragia involucrata stem was washed free of adhering foreign particles with running tap water and cut into small pieces $(0.6-0.8 \mathrm{~cm})$. Stem samples were surface sterilized by $70 \%$ alcohol, $0.5 \%$ sodium hypochlorite followed by washing thrice with sterile distilled water. The samples were inoculated in PDA medium supplemented with chloramphenicol (150mg/L)and incubated at $(28 \pm 1){ }^{\circ} \mathrm{C}$ for seven days (Higginbotham et al., 2013).

Microscopic observation of endophytic fungi.

Colony characters of pure cultures of isolated endophytic fungi were studied and observed under the Inverted Microscope (Magnus, INVI, Japan.).

Preparation of fungal fermentation broth and Extraction of secondary metabolites from the endophytic fungal culture

Extraction of secondary metabolites was carried according to the procedure described by Higginbotham et al., (2013) with slight modification. The liquid culture with evident growth of the fungus was extracted with an equal volume of ethyl acetate $(100 \%)$. The homogenate was filtered and extracted twice with ethyl acetate. The ethyl acetate extracts of $P$. citrinum CGJ-C1, $P$. citrinum CGJ-C2, Cladosporium sp. CGJ-D1 and C. hypophloia CGJD2 were labeled as EF1, EF2, EF3, and EF4 respectively.

\section{Antimicrobial Activity}

Bacterial cultures used in the present studies were obtained from microbial type culture collection (MTCC) and yeast was isolated from coffee effluent and submitted to GenBank (Dr. Anuappaiah). The bacterial strains were Escherichia coli (MTCC 118), Staphylococcus aureus (FRI722), Bacillus cereus (F4433), Micrococcus luteus (ATCC9341), Listeria monocytogenes Scott A and reference strains of Pichia kudriviavzeviiP1 (KC841145), Pichia kudriviavzevii P2(KC841146), Pichia kudriviavzevii P3 (KC841147), Candida tropicalis P4 (KC841148).

Antimicrobial activity of the ethyl acetate extract of the four-endophytic fungi was tested by disc diffusion method. The plates containing brain heart infusion medium (Himedia, India) and yeast extract peptone dextrose medium were inoculated with a $24 \mathrm{~h}$ grown inoculum of bacteria and yeast respectively. Paper discs impregnated with $200 \mu \mathrm{g}$ of endophytic fungal extracts were placed on the bacteria and yeast inoculated plates. The plates were incubated at $37^{\circ} \pm 2^{\circ} \mathrm{C}$ for bacteria and at $28^{\circ} \mathrm{C}$ for yeasts for $24 \mathrm{~h}$. The diameter of the zone of inhibition was measured ( $\mathrm{mm})$. Standard Ampicillin sodium salt $(20 \mu \mathrm{g})$ was used for antibacterial activity and Nystatin (50 $\mu \mathrm{g} / \mathrm{disc})$ was used for the antifungal activity(Arora and Kaur, 1999; Supaphon et al., 2013).
Collection and identification of Larvae of $C$. Quinquefasciatus.

Larvae (Culex quinquefasciatus) were collected from the stagnant water areas of Kodagu District, Karnataka, India (The Global Positioning System (GPS) locations were $12.46700 \mathrm{~N} 75.96700 \mathrm{E}$ ) in rectangular trays at an average temperature of $24^{\circ} \mathrm{C} \pm 2^{\circ} \mathrm{C}$. The identification of morphological characters of larvae was determined by comparing with the literature (Darsie and SamanidouVoyadjoglou, 1997; "Genus Culex - Florida Medical Entomology Laboratory,” n.d.)

\section{Larvicidal activity}

Larvicidal activity of the endophytic fungal extract was carried out according to WHO protocol with slight modifications (Matasyoh et al., 2011). The crude extracts of endophytic fungi were dissolved in dimethylsulphoxide (DMSO, analytical grade, Merck) and diluted to the required concentration with distilled water. The concentration of DMSO was kept below $1 \%$. The colonies of larvae were maintained in plastic trays containing tap water. The experiments were carried out at $27 \pm 2^{\circ} \mathrm{C}$ and $75-85 \%$ relative humidity under $14: 10$ $\mathrm{h}$ light and dark cycles. The bioassays were performed with third instar larvae of Culex quinquefasciatus and carried out in triplicate using 20 larvae for each replicate assay. The larvae were placed in $50 \mathrm{~mL}$ disposable plastic cups containing $15 \mathrm{~mL}$ of the test solution and were fed a diet of Brewer's yeast, dog biscuits, and algae in a ratio of $3: 1: 1$, respectively. The number of larvae surviving at the end of 12, 24, 36, 48 and $60 \mathrm{hrs}$ was recorded and the percentage mortality was determined by using mentioned below formula.

The percentage of mortality $=$

(No. of larva dead / No. of larvae) X100

\section{Results and Discussion}

Plants protect themselves from the pests and pathogens by symbiotically associating with the endophytes where plants provide the shelter to endophytes and in turn endophytes protect the plants against the pathogens. Endophytes with defensive secondary metabolites are selected by the host (Katoch et al., 2014). Antimicrobial secondary metabolites from microorganisms have several advantages such as no destruction of resources, sustainable use, large scale industrial productions and quality control (Liang et al., 2012). In the present study, we have isolated four endophytic fungi namely, $P$. citrinum CGJ-C1 (GenBank No. KT780618), P. citrinum CGJ-C2 (GenBank No. KP739821), Cladosporium sp. CGJ-D1 (GenBank No. KP739822), and C. bypophloia CGJ-D2 (GenBank No. KT780619). Each fungus was extracted with ethyl acetate and the characteristics of the extracts are given in Table1. Ethyl acetate extract of endophytic fungi (EF) showed a different level of inhibition of pathogenic bacteria and fungi. EF2 showed broad spectrum activity against tested organisms than other extracts. EF3 was mild antibiotic against the tested organisms. However, the extent of antimicrobial activity of other two extracts was in between the EF2 and EF3 
(Table 2). The different level of antimicrobial activity of Table 1: Characteristics of the ethyl acetate extracts of the fungal extracts may be attributed the structure and composition of chemicals present in that fungus (Teke et al., 2011). Our study is in agreement with the earlier studies on endophytic fungi containing secondary metabolites with different level of antimicrobial activity (Teke et al., 2011).

fungi isolated from T.involucrata

\begin{tabular}{ccccc}
\hline $\begin{array}{c}\text { Fungal } \\
\text { extract }\end{array}$ & $\begin{array}{c}\text { \% of yield } \\
(\mathbf{g})\end{array}$ & Color & Odour & Nature \\
\hline EF1 & 0.13 & Light orange & Characteristic & Crystal \\
EF2 & 0.10 & Yellow & Characteristic & Crystal \\
EF3 & 0.14 & Colourless & Characteristic & Crystal \\
EF4 & 0.11 & Brown & Characteristic & Sticky \\
\hline
\end{tabular}

Table 2: Antimicrobial activity of ethyl acetate extracts of endophytic fungi.

\begin{tabular}{lcccccc}
\hline \multirow{2}{*}{ Name of the organism } & \multicolumn{5}{c}{ Zone of inhibition (mm) (Mean \pm standard Deviation) } \\
\cline { 2 - 7 } & EF1 & EF2 & EF3 & EF4 & NYS & AP \\
\hline E.coli & $10 \pm 0.12$ & $10 \pm 2.1$ & NS & NS & NT & NT \\
S. aureus & NS & NS & $9 \pm 0.25$ & $12 \pm 0.76$ & NT & NT \\
B.cereus & NS & NS & NS & $12 \pm 1.43$ & NT & NT \\
M.luteus & $11 \pm 0.23$ & $12 \pm 3.0$ & $11 \pm 0.83$ & $11 \pm 1.22$ & NT & NT \\
L.monocytogenes & $10 \pm 0.11$ & NS & NS & $13 \pm 2.11$ & NT & NT \\
P.kudriviavzeviil(P1) & $11 \pm 0.98$ & $12 \pm 0.41$ & NS & NS & $18 \pm 2.39$ & $14 \pm 0.43$ \\
P. kudriviavzevii (P2) & $8 \pm 1.20$ & $10 \pm 0.17$ & $9 \pm 0.56$ & NS & $18 \pm 1.43$ & $15 \pm 0.56$ \\
P. kudriviavzevii (P3) & $8 \pm 0.32$ & $10 \pm 0.67$ & $9 \pm 1.15$ & NS & $18 \pm 2.32$ & $15 \pm 1.32$ \\
C. tropicalis (P4) & NS & $9 \pm 0.42$ & $9 \pm 1.61$ & $9 \pm 0.76$ & $24 \pm 0.98$ & $13 \pm 1.76$ \\
\hline
\end{tabular}

NYS- Nystatin; AP-Ampicillin; NS-Not Sensitive; NT-Not tested. $(\mathrm{n}=3)$

Table 3: Percentage mortality of mosquito larva (Culex Quinquefasciatus) by EF.

\begin{tabular}{|c|c|c|c|c|c|c|}
\hline \multirow{3}{*}{ EF } & & \multicolumn{5}{|c|}{ Larvicidal activity of endophytic fungal extract } \\
\hline & No hours & \multicolumn{5}{|c|}{$\%$ Mortality *at different concentration $(\mathrm{In}$ ppm) $(\%$, Mean \pm standarddeviation) $(\mathrm{n}=3)$} \\
\hline & & 100ppm & 200ppm & 300ppm & 400ppm & 500ppm \\
\hline \multirow[t]{5}{*}{ EF1 } & 12 & $0 \pm 0$ & $5 \pm 2.35$ & $38.33 \pm 2.35$ & $78.33 \pm 2.35$ & $91.66 \pm 2.35$ \\
\hline & 24 & $0 \pm 0$ & $36.66 \pm 2.35$ & $61.66 \pm 2.35$ & $90 \pm 4.08$ & $100 \pm 0$ \\
\hline & 36 & $11.66 \pm 2.35$ & $86.66 \pm 2.35$ & $90 \pm 0$ & $100 \pm 0$ & $100 \pm 0$ \\
\hline & 48 & $16.66 \pm 2.35$ & $95 \pm 4.08$ & $100 \pm 0$ & $100 \pm 0$ & $100 \pm 0$ \\
\hline & 60 & $21.66 \pm 2.35$ & $93.33 \pm 2.35$ & $100 \pm 0$ & $100 \pm 0$ & $100 \pm 0$ \\
\hline \multirow[t]{5}{*}{ EF2 } & 12 & $13.33 \pm 2.35$ & $13.33 \pm 2.35$ & $31.66 \pm=2.35$ & $61.66 \pm 2.35$ & $70 \pm 0$ \\
\hline & 24 & $31.66 \pm 2.35$ & $31.665 \pm 2.35$ & $55 \pm 0$ & $80 \pm 4.08$ & $86.66 \pm 2.35$ \\
\hline & 36 & $33.3 \pm 2.35$ & $40 \pm 0$ & $60 \pm 4.08$ & $81.66 \pm 2.35$ & $90 \pm 0$ \\
\hline & 48 & $43.33 \pm 2.35$ & $41.66 \pm 2.35$ & $65 \pm 6.23$ & $81.66 \pm 2.35$ & $98.33 \pm 2.35$ \\
\hline & 60 & $55 \pm 0$ & $61.66 \pm 2.35$ & $68.33 \pm 2.35$ & $83.3 \pm 2.35$ & $100 \pm 0$ \\
\hline \multirow[t]{5}{*}{ EF3 } & 12 & $5 \pm 2.35$ & $38.33 \pm 2.35$ & $60 \pm 4.08$ & $81.66 \pm 2.35$ & $100 \pm 0$ \\
\hline & 24 & $15 \pm 0$ & $88.33 \pm 6.23$ & $91.66 \pm 2.35$ & $83.33 \pm 2.35$ & $100 \pm 0$ \\
\hline & 36 & $15 \pm 0$ & $93.331 \pm 2.35$ & $95 \pm 4.08$ & $100 \pm 0$ & $100 \pm 0$ \\
\hline & 48 & $16.66 \pm 2.53$ & $95 \pm 0$ & $96.65 \pm 2.35$ & $100 \pm 0$ & $100 \pm 0$ \\
\hline & 60 & $30 \pm 4.08$ & $98.33 \pm 2.35$ & $100 \pm 0$ & $100 \pm 0$ & $100 \pm 0$ \\
\hline \multirow[t]{5}{*}{ EF4 } & 12 & $0 \pm 0$ & $6.66 \pm 2.35$ & $28.33 \pm 2.35$ & $35 \pm 4.08$ & $46.66 \pm 2.35$ \\
\hline & 24 & $6.66 \pm 2.35$ & $15 \pm 4.08$ & $35 \pm 4.08$ & $36.66 \pm 2.35$ & $48.33 \pm 2.35$ \\
\hline & 36 & $41.66 \pm 2.35$ & $35 \pm 0$ & $43.33 \pm 2.35$ & $80 \pm 4.08$ & $91.66 \pm 2.35$ \\
\hline & 48 & $50 \pm 4.08$ & $61.66 \pm 2.35$ & $61.66 \pm 2.35$ & $85 \pm 4.08$ & $93.33 \pm 6.23$ \\
\hline & 60 & $63.3 \pm 2.35$ & $63.33 \pm 2.35$ & $66.66 \pm 2.35$ & $93.33 \pm 2.35$ & $95 \pm 4.08$ \\
\hline
\end{tabular}

Table 4: \% Mortality of larva (Culex Quinquefasciatus) by EF.

\begin{tabular}{cccccc}
\hline $\begin{array}{c}\text { Endophytic } \\
\text { fungal extracts }\end{array}$ & $\begin{array}{c}\text { Period of } \\
\text { Treatment }\end{array}$ & LC $_{50}(\mathbf{p p m})$ & $\begin{array}{c}\text { LC } \\
\text { (ppm) }\end{array}$ & Regression & $\boldsymbol{R}^{\mathbf{2}}$ \\
\hline EF1 & 12 & 26.74 & 126.976 & $\mathrm{Y}=25.665 \mathrm{x}-34.331$ & 0.95 \\
& 24 & 14.84 & 71.952 & $\mathrm{Y}=25.334 \mathrm{x}-18.338$ & 0.97 \\
& 36 & 4.68 & 38.436 & $\mathrm{Y}=19.002 \mathrm{x}+20.658$ & 0.64 \\
& 48 & 3.05 & 31.374 & $\mathrm{Y}=17.168 \mathrm{x}+30.828$ & 0.75 \\
EF2 & 60 & 2.66 & 30.814 & $\mathrm{Y}=16.335 \mathrm{x}+33.993$ & 0.56 \\
& 12 & 42.18 & 500.6964 & $\mathrm{Y}=16.167 \mathrm{x}-10.505$ & 0.92 \\
& 24 & 12.911 & 161.466 & $\mathrm{Y}=15.834 \mathrm{X}+9.494$ & 0.93 \\
& 36 & 9.88 & 130.320 & $\mathrm{Y}=15.506 \mathrm{x}+14.474$ & 0.97 \\
& 48 & 6.91 & 99.504 & $\mathrm{Y}=15 \mathrm{x}+20.996$ & 0.94 \\
EF3 & 60 & 2.410 & 86.747 & $\mathrm{Y}=11.164 \mathrm{x}+40.166$ & 0.95 \\
& 12 & 14.88 & 82.599 & $\mathrm{Y}=23.33 \mathrm{x}-13.001$ & 0.98 \\
& 24 & 4.25 & 47.990 & $\mathrm{Y}=16.5 \mathrm{x}+26.114$ & 0.57 \\
& 36 & 3.54 & 34.055 & $\mathrm{Y}=17.667 \mathrm{x}+27.665$ & 0.57 \\
& 48 & 3.17 & 32.622 & $\mathrm{Y}=17.168 \mathrm{x}+30.158$ & 0.55 \\
& 60 & 1.619 & 27.248 & $\mathrm{Y}=14.167 \mathrm{x}+43.165$ & 0.51 \\
& 12 & 179.84 & 4817.449 & $\mathrm{Y}=12.166 \mathrm{x}-13.168$ & 0.97 \\
& 24 & 158.06 & 7138.800 & $\mathrm{Y}=10.5 \mathrm{x}-3.17$ & 0.95 \\
& 11.30 & 178.394 & $\mathrm{Y}=14.5 \mathrm{x}+14.83$ & 0.80 \\
& 36 & 3.16 & 120.073 & $\mathrm{Y}=11 \mathrm{x}+37.33$ & 0.92 \\
& 48 & 1.19 & 86.834 & $\mathrm{Y}=9.34 \mathrm{x}+48.304$ & 0.81 \\
\hline
\end{tabular}


Mosquitoes and its associated microorganisms are responsible for various diseases in people living in tropical regions (Bhattacharya and Chandra, 2014). Antimosquito medicines derived from natural products were well received by the people due to easy biodegradation and less side effects (Rajasekaran and Duraikannan, 2012). In our study, the endophytic extracts showed a moderate to a significant level of larvicidal activity. All the extracts were active against larva even at $100 \mathrm{ppm}$ with EF4 extract showing the highest activity. The toxicity towards the larva was showing a positive relation with the time of incubation and concentration of EF.100 percent larval death was observed in all the extract when the concentration was scaled up to $500 \mathrm{ppm}$. All the larvae were almost dead at 500ppm in EF1 and EF3 extract after $12 \mathrm{~h}$ treatment (Table 3). The EF3 extract showed a significant larvicidal activity with anLC $\mathrm{C}_{50}$ and $\mathrm{LC}_{90}$ values of 4.25 and $47.990 \mathrm{ppm}$ (at 24h) respectively (Table 4). The extent of the larvicidal potential of EF3 was highest among the ethyl acetate extracts of the endophytes. Interestingly, Bhattacharya and Chandra (2011) have studied the larvicidal activity of T. involucrata extract (Bhattacharya and Chandra, 2014). However, the larvicidal potency of the endophytic extract was even better than the T. involucrata extract. Our study supports the view that the endophytes share the bioactive properties of the host plants as put forwarded by Zhao et al., (2010). The endophytic extract in crude form or the bioactive principle present in it can be sprayed on the stagnant sewage water which is the breeding ground for mosquitoes. But understanding the mechanism of action of the leads present in the extract will help in designing the better strategy for mosquito menace. The endophytic extract of $T$. involucrata showed better activity than the extracts of endophytes of different plants (Matasyoh et al., 2011) as well as some medicinal plants reported earlier (Bhattacharya and Chandra, 2014).

\section{Conclusion}

Overall the present study for the first time established the possible role of endophytes of T. involucrata in the development of antimicrobial and larvicidal agents. All the endophytic extracts selected in this study showed varying degree of bioactivity. Further studies on the isolation, characterization and understanding the mechanism of action of lead molecules will aid in the formulation of a novel as well as cost effective drugs against microbe-caused diseases.

\section{References}

1. Arora DS, Kaur J. Antimicrobial activity of spices. Int. J. Antimicrob. Agents. 12, (1999): 257-262. doi:10.1016/S0924-8579(99)00074-6.

2. Bhattacharya K, Chandra G. Phagodeterrence, larvicidal and oviposition deterrence activity of Tragia involucrata L.(Euphorbiaceae) root extractives against vector of lymphatic filariasis Culex quinquefasciatus (Diptera: Culicidae). Asian Pac. J. Trop. Dis. 4, (2014): S226-S232.

3. Cui J, Guo S, Xiao P. Antitumor and antimicrobial activities of endophytic fungi from medicinal parts of
Aquilaria sinensis. J. Zhejiang Univ. Sci. B 12, (2011): 385392.

4. Darsie RF, Samanidou-Voyadjoglou A. Keys for the identification of the mosquitoes of Greece. J. Am. Mosq. Control Assoc. 13, (1997): 247-254.

5. Farook SM, Atlee WC. Antidiabetic and hypolipidemic potential of Tragia involucrata Linn. In streptozotocinnicotinamide induced type II diabetic rats. Int. J. Pharm. Pharm. Sci. 3, (2011): 103-109.

6. Genus Culex - Florida Medical Entomology Laboratory [WWWW Document], n.d. URL http://fmel.ifas.ufl.edu/key/genus/culex_quin.shtml (accessed 8.20.16).

7. Higginbotham SJ, Arnold AE, Ibañez A, Spadafora C, Coley PD, Kursar TA. Bioactivity of fungal endophytes as a function of endophyte taxonomy and the taxonomy and distribution of their host plants. PloS One 8, (2013): e73192.

8. Joshi CG, Gopal M, Byregowda SM. Cytotoxic activity of Tragia involucrata Linn. extracts. Am.-Eurasian J. Toxicol. Sci. 3, (2011a): 67-69.

9. Joshi CG, Gopal M, Kumari NS. Antitumor activity of hexane and ethyl acetate extracts of Tragia involucrata. Int. J. Cancer Res. 7, (2011b): 267-277.

10. Joshi GC, Gopal M. Antifertility Activity of Hexane and Ethyl Acetate Extracts of Aerial Parts of Tragia involucrata. Linn. J. Pharmacol. Toxicol. 6, (2011): 548-553. doi:10.3923/jpt.2011.548.553

11. Katoch M, Singh G, Sharma S, Gupta N, Sangwan PL, Saxena AK. Cytotoxic and antimicrobial activities of endophytic fungi isolated from Bacopa monnieri (L.) Pennell (Scrophulariaceae). BMC Complement. Altern. Med. 14, (2014): 52. doi:10.1186/1472-6882-14-52

12. Liang $\mathrm{H}$, Xing $\mathrm{Y}$, Chen J, Zhang D, Guo S, Wang C. Antimicrobial activities of endophytic fungi isolated from Ophiopogon japonicus (Liliaceae). BMC Complement. Altern. Med. 12, (2012): 1.

13. Matasyoh JC, Dittrich B, Schueffler A, Laatsch H. Larvicidal activity of metabolites from the endophytic Podospora sp. against the malaria vector Anopheles gambiae. Parasitol. Res. 108, (2011): 561-566. doi:10.1007/s00436010-2098-1

14. Rajasekaran A, Duraikannan G. Larvicidal activity of plant extracts on Aedes Aegypti L. Asian Pac. J. Trop. Biomed. 2, (2012): S1578-S1582. doi:10.1016/S2221-1691(12)60456-0

15. Strobel G, Daisy B. Bioprospecting for Microbial Endophytes and Their Natural Products. Microbiol. Mol. Biol. Rev. 67, (2003): 491-502. doi:10.1128/MMBR.67.4.491-502.2003

16. Supaphon P, Phongpaichit S, Rukachaisirikul V, Sakayaroj J. Antimicrobial Potential of Endophytic Fungi Derived from Three Seagrass Species: Cymodocea serrulata, Halophila ovalis and Thalassia hemprichii. PLOS ONE 8, (2013): e72520. doi:10.1371/journal.pone.0072520 
17. Tamokou J de D, Chouna JR, Fischer-Fodor E, Chereches G, Barbos O, Damian G, Benedec D, Duma M, Efouet APN, Wabo HK, Kuiate JR, Mot A, Silaghi-Dumitrescu R. Anticancer and Antimicrobial Activities of Some Antioxidant-Rich Cameroonian Medicinal Plants. PLoS ONE 8. (2013): doi:10.1371/journal.pone.0055880

18. Teke GN, Lunga PK, Wabo HK, Kuiate J-R, Vilarem G, Giacinti G, Kikuchi H, Oshima Y. Antimicrobial and antioxidant properties of methanol extract, fractions and compounds from the stem bark of Entada abyssinica Stend ex A. Satabie. BMC Complement. Altern. Med. 11, (2011): 57. doi:10.1186/1472-6882-11-57

19. Zhao J, Zhou L, Wang J, Shan T, Zhong L, Liu X, Gao X. Endophytic fungi for producing bioactive compounds originally from their host plants. Curr Res Technol Educ Trop Appl Microbiol Microb. Biotechnol 1, (2010): 567576.

\section{Cite this article as:}

Ananda Danagoudar, Chandrashekhar G. Joshi, Nivya M.T., Manjunath H.M., Jagadeesha Poyya and Sunil Kumar R. Antimicrobial and Larvicidal potential of Endophytic fungi isolated from Tragia involucrata Linn. Annals of Plant Sciences 6.01 (2017): 1494-1498. DOI: http://dx.doi.org/10.21746/aps.2017.01.002

Source of support: University Grant Commission (UGC)F.No.42-671/2013 (SR), New Delhi, India

Conflict of interest: Nil 